EPJ Web of Conferences 101, 06002 (2015)

DOI: $10.1051 /$ epjconf/ 201510106002

(C) Owned by the authors, published by EDP Sciences, 2015

\title{
The CoRoT Bright Star Catalogue
}

Annie Baglina, Eric Michel, and the CoRoT Team

LESIA, Observatoire de Paris, 5 pl. J. Janssen, 92195 MEUDON, France

\begin{abstract}
The CoRoT bright stars catalogue describes the 143 different objects observed during the mission, in the "bright star" field of CoRoT, and their major properties. The almost continuous observations lasted between 20 and 156 days, with a regular 32s sampling time.This catalogue, as well as the corresponding data, are available at CDS (Centre de données astronomiques de Strasbourg) in Vizier.
\end{abstract}

\section{The CoRoT "Bright Stars"}

The CoRoT "bright stars" programme was focussed essentially on astroseismology. It has been optimized for stars with $5.4 \leq m V \leq 9.5$ and the instrument was designed to be able to detect solar like oscillations in a reasonable sample of targets. It measures the stellar photon flux in the visible with a high precision, close to the photon noise limit: for a 6th magnitude star the noise level is of $0.156 \mathrm{ppm}^{2} / \mu \mathrm{Hz}$. Due to the constraints on the mission, only two regions of the sky allow continuous observations during long periods: the "CoRoT eyes", approximately two disks of 10 degrees radius centered at 0 deg declination, $6 \mathrm{~h} 50$ and $18 \mathrm{~h} 50$ hour angles. [1].

The observations from space have been performed between January 2007 and November 2012 .

Among the 143 different stars observed with CoRoT, 15 have been observed twice and one has been observed three times. They have spectral types from $\mathrm{O}$ to $\mathrm{G}$, and luminosity classes from $\mathrm{V}$ to III. Their position in the HR diagram is shown in Fig.1.

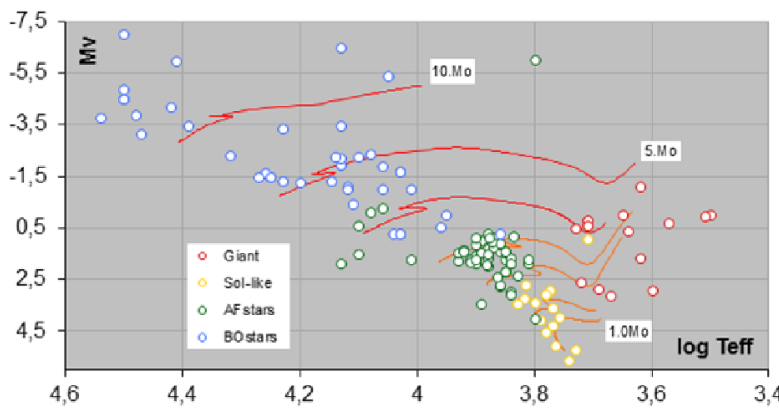

\begin{tabular}{|c|r|l|l|l|l|l|l}
\hline HD & \multicolumn{1}{|c|}{ CID } & Start Time & length & Star type & SpT & mV & Remarks \\
\hline 181420 & 8652 & $09 / 05 / 2007$ & 158 & FGK Sol-like & F2 & 6.57 & \\
\hline 181906 & 8777 & $09 / 05 / 2007$ & 158 & FGK Sol-like & F8 & 7.65 & \\
\hline 181907 & 8774 & $09 / 05 / 2007$ & 158 & Giant & G8 III & 5.83 & \\
\hline 49808 & 1 & $15 / 10 / 2007$ & 138 & A/early F & FOV & 7.98 & \\
\hline 49294 & 29 & $15 / 10 / 2007$ & 138 & D Scut & A2 & 7.0 & \\
\hline 49862 & 79 & $15 / 10 / 2007$ & 138 & G Dor & A5 & 9.47 & \\
\hline 50064 & 14 & $15 / 10 / 2007$ & 138 & B & B6I & 8.29 & \\
\hline 50230 & 44 & $15 / 10 / 2007$ & 138 & B Ceph & B3 & 8.95 & \\
\hline 49330 & 49 & $15 / 10 / 2007$ & 138 & Be & B0.5IVe & 8.88 & \\
\hline 50209 & 26 & $15 / 10 / 2007$ & 138 & Be & B8IVe & 8.39 & \\
\hline 49385 & 18 & $15 / 10 / 2007$ & 138 & FGK Sol-like & G0 & 7.89 & OST \\
\hline 49933 & 20 & $15 / 10 / 2007$ & 138 & FGK Sol-like & F2 & 5.77 & OST \\
\hline 49434 & 100 & $15 / 10 / 2007$ & 138 & G Dor & F1 V & 5.75 & \\
\hline 48784 & 3619 & $03 / 03 / 2008$ & 28 & A/early F & F0 & 6.66 & \\
\hline
\end{tabular}

Fig. 1. Right: HR diagram of the bright stars observed with CoRoT. $M_{\mathrm{v}}$ and $T_{\text {eff }}$ after [3]. Left: A few lines of the CoRoT Bright Star Catalogue.

The wide range of the targets, as well as the long uninterrupted observations, has allowed to detect a large variety of behaviors some expected, some unexpected. A few examples are mentioned below.

HD 52265, a bright G Main Sequence solar like pulsator with a known planet, shows solar-type pulsations; frequencies and amplitudes have been measured with a very high accuracy [2] as well as

a e-mail: annie.baglin@obspm.fr

This is an Open Access article distributed under the terms of the Creative Commons Attribution License 4.0, which permits unrestricted use, distribution, and reproduction in any medium, provided the original work is properly cited. 
the inclination of its spin axis [4], putting severe constraints on the mass, radius and age of the host star, and also infering a more precise mass of the companion confirming that it is a $1.17-1.26 M_{\text {Jup }}$ planet and not a brown dwarf [7] .

HD 49330, a Be star which has been catched by chance during an outburst, shows correlation between the pulsation amplitudes and the different outburst phases [5]. The amplitude of the p-modes decrease during the burst and an the energy in very low frequency peaks increases. This is interpreted as due to the stochastic excitation of gravito-inertial modes by the structural perturbation induced in surface layers when mater is ejected and transmitted to the decretion disk [9].

HD181907 is the first red giant showing a complete spectrum of solar like oscillations. The echelle diagram revealed the presence of non-radial pulsations $(1=1,2)[6]$. These oscillations, quite common in red giants, are now used to probe the galactic structure [10]. In addition a sharp structure in the interior has been detected and attributed to the local depression of the sound speed in the Helium ionisation zone [8].

But, beyond the results already published, many more discoveries are certainly hidden in the data!

\section{The content of the catalogue}

For all stars of this catalogue, CoRoT light curves with 32s regular sampling (in solar system barycentric time scale) are available with various length between 20 and 158 days at CDS, in the Vizier environment.

The catalogue contains 159 lines, one per object for each epoch of observation.

For each line the following fields are proposed:

-1 HD (HD number when available), -2 CID (CoRoT identifier), -3 Start time (starting UTC date), -4 Length (duration of the observations, in days), -5 Star type (see below), -6 Sp T (spectral type and luminosity class) [3] -7 mV [3], - 8 Remarks : EB (eclipsing binary); OST (observed several times) ....

The star types are defined as:

O : O type stars, B : B type stars, Be : B type stars with emission lines, B Ceph : Beta Cephei stars*, CP : Chemically peculiar stars*, A/early F : A and early F type stars, D Scut : Delta Scuti stars*, G Dor : Gamma Doradus stars*, Late F/G : Late F and G stars, FGK Sol-like : F, G, K type stars showing solar-like pulsations*, KM : K or M type stars, Giant : Red giants (occasionally yellow), TBC : to be confirmed, where * means : previously known or discovered with CoRoT data.

The CoRoT space mission, launched on 2006 December 27, was developed and operated by the Centre National d'Etudes Spatiales (CNES, France) with the participation of the Science Program of the European Space Agency (ESA), the ESA Research and Scientific Support Department (RSSD), Austria, Belgium, Brazil, Germany and Spain.

\section{References}

1. Auvergne, A. et al,. A\&A 506, (2009) 411

2. Ballot, J., Gizon, L., Samadi, R. et al., A\&A 530, (2011) 97

3. Charpinet, S., Cuvilo, J., Platzer, J. et al., ESA-SP 1306,(2006) 353

4. Gizon, L., Ballot, J., Michel, E. et al., PNAS 110,(2013) 11013267

5. Huat, A.-L., Hubert, A.-M., Baudin,F. et al., A\&A 506, (2009) 95

6. Carrier, F., De Ridder, J., Baudin, F. et al., A\&A 509, (2010) 73

7. Lebreton,Y., Goupil, M-J. A\&A 569 ,(2014) 21

8. Miglio, A., Montalban, J., Carrier, F. et al., A\&A 520, (2010) 6

9. Neiner, C., Mathis, S., Saio, H., Lee, U., ASPC 479, (2013) 319

10. Miglio, A., Girardi, L., Rodrigues, Thaise S., Stello, D., Chaplin, William J., ASSP 39, (2015) 11 